\section{Viral encephalitis and virus-mediated memory disorders}

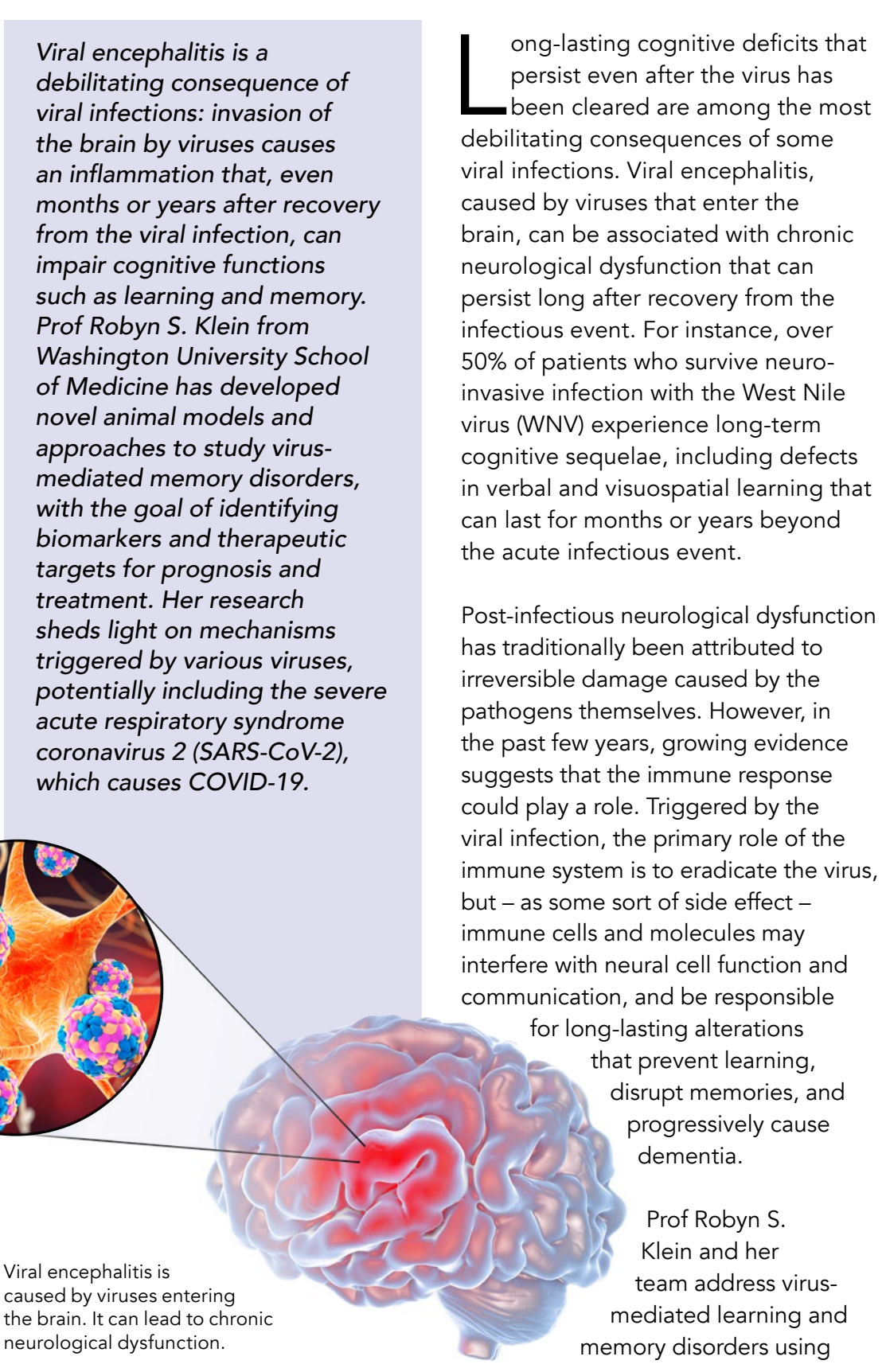

tools and technologies that combin basic neuroscience and immunology

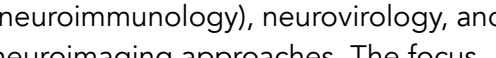
of their work has been on emerging viral infections, which include West Nile (WNV) and Zika (ZIKV) viruses. WNV and ZIKV both target neurons and are associated with post-infectious cognitive dysfunction.

DURING THE ACUTE STAGE When viruses invade the brain, they trigger the immune system, whose primarily aim is to defend the organism against all kinds of pathogens. Innate immunity is the first line of defence. Unlike adaptive immunity (the other component of the immune system), it is not specific. the innate immune defence mechanisms involved in response to nature of the pathogen

Inflammation is one of the main responses of innate immunity. All types of brain cells (neurons, astrocytes, oligodendrocytes and microglia). express immune molecules that induce the recruitment of virus-specific cells into infected regions of the brain. The aim is to promote pathogen clearance. Mediated by cytokines (small proteins that convey signals from one cell to another), inflammation is localised and allows the site of infection within the brain to be identified and cleared of the virus.

Clinical signs arise during inflammation and are indicators of acute infection.

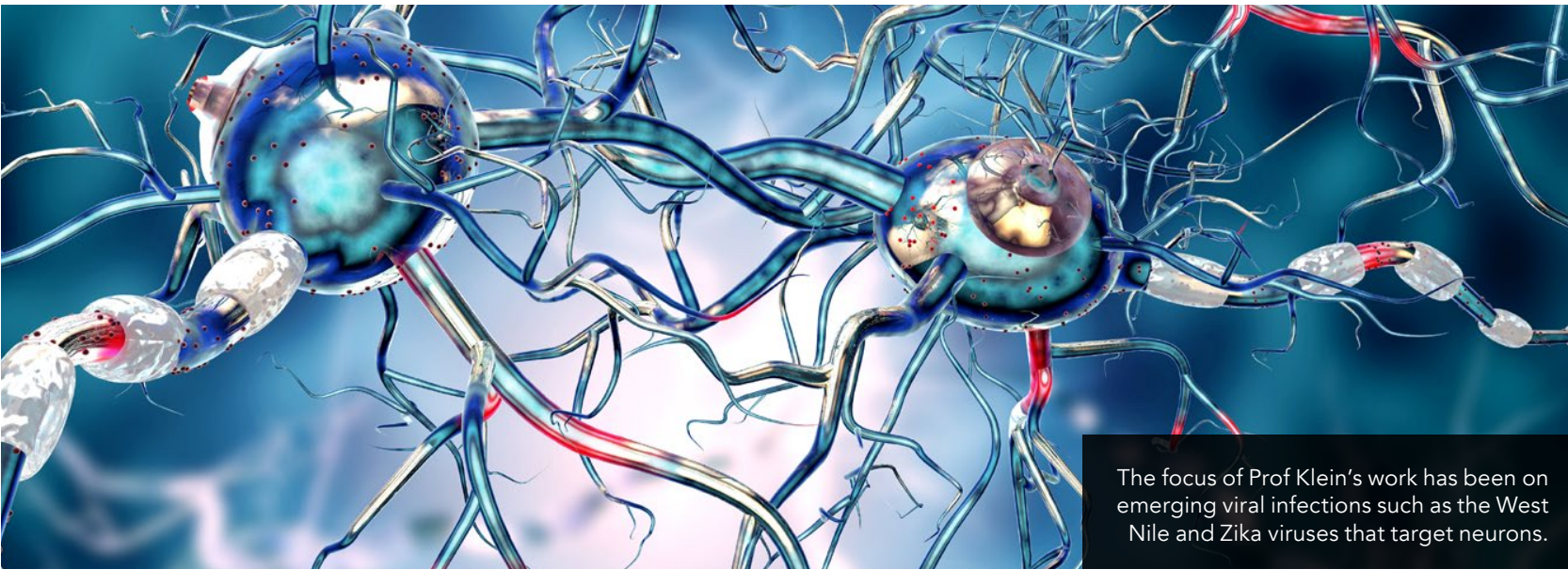

daptive changes in behaviou, including fatigue, hypersomnia decreased activity and social interactions, and an inability to concentrate. The purpose of these promote survival. Sometimes, howe in the most severe cases of infection, inflammatory responses may progress to neurological deficits, delirium, or even coma.

linked to ongoing inflammatory processes: inflammation, acutely induced by immune responses to pathog, pesists eren after the pathogen was elminated. Recruited may affect cells or regions other tha those initilly infected. This leads to chronic complications because, while the immune system plays an essentia role in eliminating the pathogen, the cytokines released by immun

CHRONIC INFLAMMATORY cells have multiple roles and their PROCESSES activities may therefore disrupt norm brain functioning. mpaired learning or memory disorders are examples of symptoms that can arise months or even years after recovery from an

acute infection. They are clinical signs of post-infectious, chronic inflammatory procin

While the immune system plays an essential role in eliminating the pathogen, the cytokines released by immune cells have multiple roles and Historically, postsequelae have been attributed to been attributed to their activities may therefore disrupt

normal brain functioning.

ing pathogen or the immune response to it during the acute stage. However, while inflammation during the acute stage is localised in brain regions infected by the virus, neurological dysfunction in patients that survive the acute infection can affect brain regions other than the ones initialy e directly caused by the virus itsel.

Chronic complications from

cells derail normal functioning of memory so that learning is impaired, The memories cytokines involved in antiviral pathways that regulate synaptic plasticity and neurogenesis, and therefore affect communication infection neurological sequelae.

T CELLS AND INTERFERON- $\gamma$ visuospatial learning and memory. fter recovery from infection with both WNV and ZIKV.

Prof Klein examined mice that recovered from WNV or ZIKV. ZIKV eplicates mostly in the hippocampus and, after recovery a loss of neurons and of postsynaptic terminals (parts of neuron that, at the level of a connection between two neurons - called synapse - receive the neural signal) can be sserved. WNV replicates throughout the entre brain and, while there is no sign of neuronal loss, a loss of inals (parts of synapses that send neural signals) was visible in mice that recovered

While WNV and ZIKV have different effects viruses can cause a

long-term cognitive decline including learning and memory deficits. This suggests that cognitive sequelae rely on a common mechanism.

Interferon- $\gamma$ (IFN- $\gamma$ ), a cytokine released by $T$ cells that stay in the brain even after recovery from infection, plays a role in targeting viruses, but also receptor (thus making IFN- $\gamma$ ineffective) do not display the typical neuronal or synaptic loss, and do
spatial learning deficits. cells (a type of pathogen-specific influences synapses. Prof Klein reported that mice lacking IFN- $\gamma$ or the IFN- $\gamma$ 
WNV Recovery: Model for synapse elimination/recovery

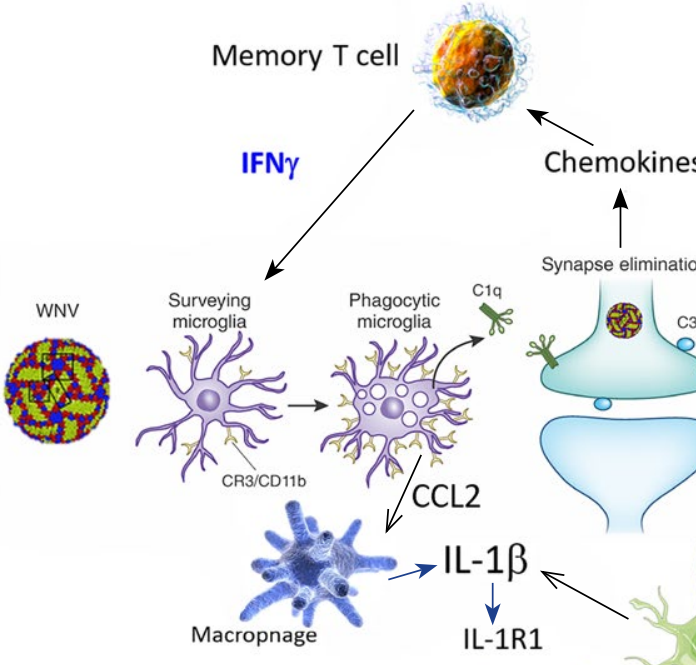

Inactivation of IL-1R1--|

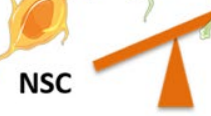

Prof Klein's research suggests that targeting IFN- $\gamma$ or IL-1 pathways, or components of the
complement system, could prevent memory disorders during recovery from vira encephalitits

N- $\gamma$ mediates synapse elimination and a similar mechanism: the complement IFN- $\gamma$ mediates synapse elimination and a similar mechanism: the complement
resulting cognitive deficits by activating system is required in the elimination microglia (resident macrophages in of presynaptic terminals by microglia. the brain). Indeed, in mice lacking the Indeed, mice with fewer microglia or IFN-y receptor, there is no increase deficiency in the complement systen in microglia activation after infection. are protected from WNV-induced Furthermore, when IFN- $\gamma$ receptor is synaptic terminal loss.

deactivated specifically in microglia, there is no synapse elimination and The research of Prof Klein's team the hins how viral infection

Prof Klein and her team study how the activities of persistent antiviral immune cells derail normal functioning of memory.

microglia are involved in IFNmediated synapse

MICROGLIA AND COMPLEMENT The complement system consists of a number of small proteins that enhance the ability of the immune system to clear pathogens. It constitutes a key component of innate immunity.

During early postnatal development, the complement system mediates synapse elimination by microglia. Pr hippocampad adut neurons induces infection, another cytokine expressed by in

One of the specificities of the

hippocampus is that adult neurogenesis happens there: adult neural stem cells evolve into either new neurons or astrocytes. This process is regulated by various factors, including local molecules, exercise, aging and inflammation. IL-1 is one of these factors, causing derailment of neurogenesis in favour of astrogenesis. IL-1 is a cytokine that regulates a type of antiviral T cell named effector T cells, and that is for example responsible for the onset of fever, which is one the immune defence mechanisms. It memory; after an injection of $1-1$, these functions are an imaced.

In the context of WNV infection, astrocytes release a high quantity of $\mathrm{L}-1$, and this results in neurogenes generating more astrocytes, instead of producing new neurons which are essential for learning and memory. Inhibiting the IL-1 pathway allows normal neurogenesis and resistance to spatial learning deficits.

Towards understanding more viruses Prof Klein's research suggests that targeting IFN- $\gamma$ or IL-1 pathways, or components of the complement system, recovery from viral encephalitis.

While most of her work was done on WNV and ZIKV, other emerging viruses activate the same mechanisms and trigger similar neurological sequelae. The team has begun examining the effects of SARS-CoV-2 on brain function, since COVID-19 has been linked to both neurological diseases and post-infectious psychiatric and cognitive impairments. Given that these mechanisms may be generalisable to multiple diseases of cognition, and that there are no treatments that reverse memory deficits, there is a pressing need to understand how viral infections lead to diseases of progressive pathologic forgetting and gradually cause

\section{Prof Robyn Klein}

E: rklein@wustl.edu T: +1 3143746614 and +1 3142862140 W: https://kleinlab.wustl.edu/

\section{Research Objectives}

Prof Klein studies virus-mediated memory disorders with the goal of identifying biomarkers and therapeutic targets for prognosis and treatment.

\section{Detail}

Bio

Robyn S. Klein received her BA with honours in Biology Nollege, Columbia University, a PhD in Meuricin Medicine at the Brigham \& Wom's fellowship in Infectious Diseases at the Massachusetts General Hospital, and post-doctoral training in Immunology, all at Harvard University. Dr Klein joined the Washington University School of Medicine (WUSM) in 2003, where she attained Professorships in the Departments of Medicine, Pathology \& Immunology, and Neuroscience. She has served in the Directorship of the WUSM Medical Scientist Training Program, as President of the WUSM Academic Women Network, and continues to mentor junior faculty through the WUSM Family-Wise Mentoring Program. Dr Klein has served as Vice Provost and Associate Dean for Graduate Education, the latter of which overseas the Division of Biology \& Biomedical Sciences, and founded

\section{Funding}

- DTRA

- NIAID

\section{Collaborators}

- Jinsheng Yu, PhD

- Beth Stevens, PhD

- Haina Shin, PhD

- Michael Diamond, MD, PhD

- Mehul Suthar, PhD

- Yongjian Liu, PhD

- Jonathon Miner, MD, PhD

WashingtonUniversityin St.Louis SCHOOL OF MEDICINE

\section{References}

Garber, C., Soung, A., Vollmer, L.L., Kanmogne, M., Last, A., Brown, J., \& Klein, R.S. (2019). T cells promote microglia-mediated synaptic elimination and cognitive dysfunction during recovery from neuropathogenic
flaviviruses. Nature neuroscience, 22(8), 1276-1288 flaviviruses. Nature neuroscience, 22(8), 1276-1288.
Available at: https://doi.org/10.1038/s41593-019-0427-y

Garber, C., Vasek, M.J., Vollmer, L.L., Sun, T., Jiang, X., \& Klein, R.S. (2018). Astrocytes decrease adult neurogenesis immunology 19(2), 151-161. Available at. https.//doi. org/10.1038/s41590-017-0021-y

Klein, R.S., Garber, C., \& Howard, N. (2017). Infectious immunity in the central nervous system and brain function. Nature immunology, 18(2), 132-141. Available at: https:// doi.org/10.1038/ni.3656

Vasek, M.J., Garber, C., Klein, R.S. et al. (2016). A complement-microglial axis drives synapse loss during vins-5uced memory imparment. Nature, 534(7608), 538-543. Available at: https://doi.org/10.1038/ nature 18283

Krukowski, K., \& Rosi, S. (2019). The dark side of antiviral T cell responses. Nature neuroscience, 22(8), 1199-1200.

\section{Personal Response}

Because of the various roles played by IFN- $y$ or IL-1, do you think it could be possible to target these pathways to prevent memory deficits without causing too many side effects?

We have treated acutely infected mice with Anakinra, an FDA-approved drug for arthritis, and demonstrated that only four days of treatment prevented the development of spatial learning defects. Thus, we know the treatment of post-infectious cognitive dysfunction. However, we have also shown that IL-1 is critical for viral clearance during acute WNV encephalitis in mice. Thus, it is possible that targeting IL-1 could limit virologic control, which could be extremely detrimental to
patients acutely infected with WNV or other viruses.
Astrocytes, star-shaped cells that assume other roles, are also involved in the development of post-infection her colleal sequelae. Prot Klin and 